\section{Percepción de conocimiento sobre las demencias en profesionales de la salud de Chile}

\section{Chilean Healthcare Professionals' Perception of Knowledge about Dementia}

\section{Sr. Editor}

El aumento de la esperanza de vida se ha asociado a un aumento exponencial de las personas con enfermedades crónicas no transmisibles, entre ellas la enfermedad de Alzheimer y otras demencias. En Chile, uno de los países mas envejecidos de Latinoamérica, más de 180 mil personas ( $1 \%$ de los chilenos) presenta algún tipo de demencia, cifra que se incrementará a más de 626.000 personas ( $3 \%$ de los chilenos) en $2050^{1}$. Las demencias constituyen la enfermedad con el mayor incremento porcentual como causa de años de vida perdidos por discapacidad o muerte prematura (más de $200 \%$ en los últimos años) y como causa de muerte, con un incremento de más de $526 \%^{2,3}$.

No obstante lo anterior, estudios internacionales sugieren que el conocimiento que tienen los profesionales de la Salud respecto de estas patologías es insatisfactorio y no se condice con el impacto en la salud pública de estas enfermedades ${ }^{4}$. En Argentina ${ }^{5}$, un estudio mostró que más de $60 \%$ de los médicos consideraba no tener suficiente conocimiento en esta área, siendo esta situación más acentuada en los médicos generales. En Chile, los curriculum de las profesiones médicas aún abordan escasamente los trastornos demenciantes y no existen datos sobre el nivel de conocimiento de los profesionales de salud.

Para conocer la realidad nacional, se dispuso un cuestionario auto-administrado, construido en base a un instrumento previo 5 . El cuestionario constaba de dos secciones, una para todos los profesionales de salud y otra exclusiva para médicos. En la sección orientada a todos los profesionales, se indagó respecto de la formación que habían recibido en el pre-grado, así como la percepción respecto de su nivel de preparación para brindar atención a un paciente con demencia. En la sección para médicos, se indagó respecto de los exámenes solicitados a las personas con sospecha de deterioro cognitivo, así como los tratamientos indicados. El cuestionario se administró mediante una encuesta on-line difundida por diferentes métodos, tales como redes sociales y correos electrónicos distribuidos por diferentes sociedades científicas.

Se realizó un análisis descriptivo de los resultados, y se calculó $\chi^{2}$ para las variables categoriales.

Se aplicaron 922 encuestas a profesionales de la salud de diferentes áreas profesionales, de las cuales 123 no se consideraron en el análisis final, debido a que se encontraban incompletas. De los 799 participantes que contestaron la encuesta (edad promedio: $35,9 \pm 10,8$ años), $37 \%$ fueron médicos (173 médicos generales y 110 médicos especialistas) y $63 \%$ otros profesionales de la salud (psicólogos, terapeutas ocupacionales, fonoaudiólogos, enfermeras). Si bien 65\% de los profesionales asistió a alguna clase de este tópico en la universidad, $51 \%$ de los encuestados consideraban que su conocimiento sobre las demencias era insuficiente y $81 \%$ considero que no se sentía preparado para atender a una persona con demencia. El nivel de preparación difiere entre diferencias entre el tipo de profesión y la percepción de preparación $\left(\chi^{2}=9.4877\right.$; $\left.p<0,05\right)$, siendo mayor la percepción de preparación en profesionales fonoaudiólogos y terapeutas ocupacionales (Figura 1).

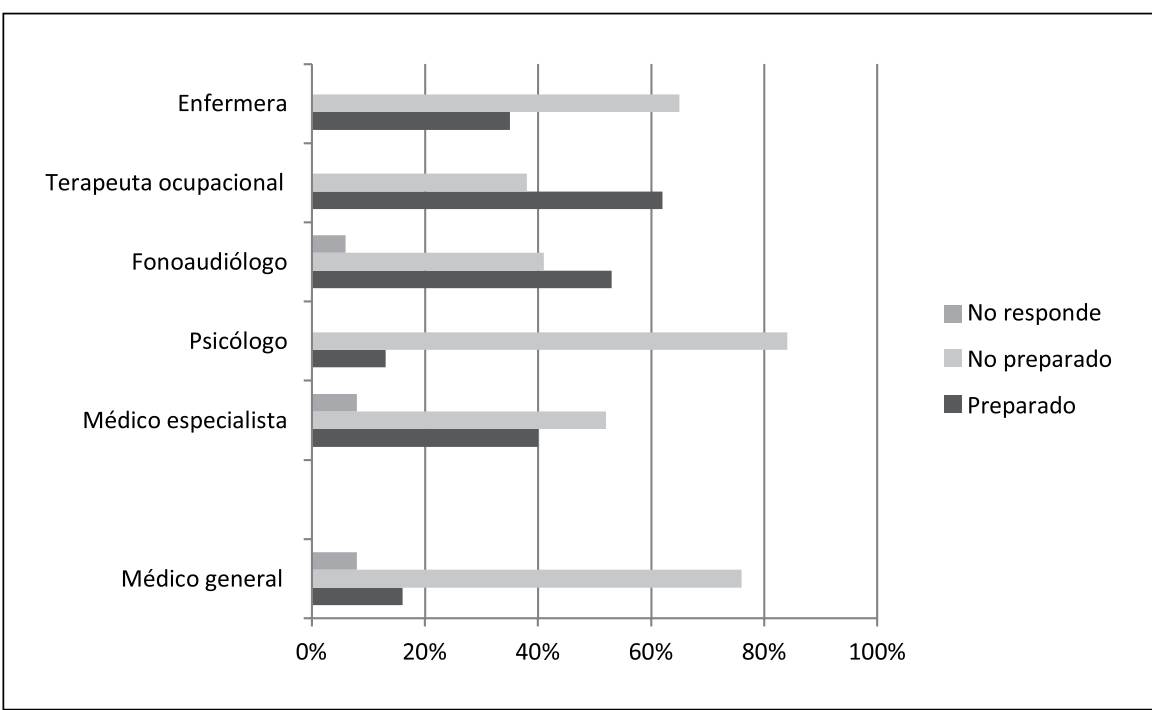

Figura 1. Percepción de Conocimiento sobre demencia en profesionales de la salud. 
Dentro del grupo de los médicos, la apreciación de sentirse "no preparados" fue más evidente en médicos generales ( $72 \%)$. El $42 \%$ de los médicos deriva a un especialista, siendo el neurólogo el profesional identificado como más idóneo para la atención de estos casos.

Estos resultados reflejan que, a pesar del incremento de la prevalencia de las demencias, existe la percepción de un nivel de preparación insuficiente en esta materia por parte de los profesionales de la salud chilenos. La falta de preparación de los médicos generales en demencia es concordante con estudios previos ${ }^{4}$ Es importante considerar que, en la mayoría de los países, es el médico general el primer punto de contacto para las personas con demencia, siendo la Atención Primaria el nivel asistencial al que habitualmente consultan ${ }^{3}$. De acuerdo a los resultados obtenidos, su conducta frente a la sospecha de este diagnóstico consiste en la derivación al especialista, lo que es otra limitante, dado que el número de especialistas en Chile es reducido, con lo que difícilmente se podría dar cobertura a la necesidad creciente de este grupo de pacientes.

La falta de reconocimiento de la enfermedad por parte de los profesionales de la salud, así como el retraso en el diagnóstico, implica a su vez que tanto enfermos como sus familias se ven privados de la posibilidad de acceder a un tratamiento óptimo y oportuno, o de recibir apoyo psicosocial ${ }^{6}$.

La baja percepción de preparación de los psicólogos/ as $(84 \%)$ y enfermeros/as $(65 \%)$ es particularmente sensible si se considera que el profesional psicólogo ocupa un rol central tanto al momento de brindar el soporte psicosocial a las familias, como en el proceso de psicoeducación para el manejo de los trastornos de conducta. Por otro lado, los profesionales de enfermería ocupan un papel fundamental en la atención de esta población tanto en atención primaria como en servicios de Medicina Interna.

Los resultados de este estudio deben ser interpretados con cautela por la metodología empleada en el reclutamiento de los encuestados, pudiendo llevar a un posible sesgo en solo aquellos profesionales con mayor interés en las demencias hayan aceptado a responderla, no obstante sus resultados ponen una nota de aviso respecto a la calidad de atención que se les está brindando actualmente a las personas con demencia y sus familias.

El año 2012 la OMS declaró a las demencias como una prioridad socio-sanitaria, llamando a los diferentes países a generar e implementar políticas públicas para abordar esta multidimensional problemática. El Ministerio de Salud de Chile constituyó en el 2014 una mesa de trabajo interministerial con participación de la sociedad civil y expertos para elaborar un plan de demencia. Los resultados de esta encuesta sugieren que la formación de los profesionales de salud en demencia debería constituir un elemento fundamental para mejorar la atención de quienes padecen la enfermedad y sus familias.

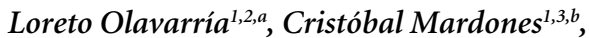
Carolina Delgado ${ }^{4,5}$, Andrea Slachevsky Ch. ${ }^{1,5,6,7,8}$ ${ }^{1}$ Corporación Profesional de Alzheimer y otras Demencias (COPRAD). Santiago, Chile. ${ }^{2}$ Unidad de Patologías Neurodegenerativas, Centro de Rehabilitación Capredena La Florida.

Santiago, Chile.

${ }^{3}$ Unidad de Medicina Física y Rehabilitación, Hospital Clínico Metropolitano La Florida. Santiago, Chile.

${ }^{4}$ Departamento de Neurología, Clínica Santa María. Santiago, Chile.

${ }^{5}$ Departamento de Fisiopatología, ICBM $y$ Departamento de Ciencias Neurológicas Oriente, Facultad de Medicina, Universidad de Chile. Santiago, Chile.

${ }^{6}$ Centro FONDAP Centro de Gerociencias. Salud Mental y Metabolismo (GERO).

${ }^{7}$ Unidad de Neurología Cognitiva y Demencias, Servicio de Neurología, Hospital del Salvador, Santiago, Chile.

${ }^{8}$ Servicio de Neurología, Departamento de Medicina Clínica Alemana-Universidad del Desarrollo, Santiago, Chile. ${ }^{a}$ Psicóloga

${ }^{b}$ Fonoaudiólogo

\section{Referencias}

1. Slachevsky A, Arriagada P, Maturana J, Rojas R. Enfermedad de Alzheimer y otras Demencias en Chile: Propuesta de un Plan Nacional de Alzheimer y otras demencias: Coprad. Sonepsyn, 2012.

2. Lozano R, Naghavi M, Foreman K, Lim S, Shibuya K, Aboyans $\mathrm{V}$, et al. Global and regional mortality from 235 causes of death for 20 age groups in 1990 and 2010: a systematic analysis for the Global Burden of Disease Study 2010. Lancet 2012; 380 (9859): 2095-128.

3. Murray CJ, Vos T, Lozano R, Naghavi M, Flaxman AD, Michaud C et al. Disability-adjusted life years (DALYs) for 291 diseases and injuries in 21 regions, 1990-2010: a systematic analysis for the Global Burden of Disease Study 2010. Lancet 2012; 380 (9859): 2197-223.

4. Pathak K, Montgomery A. General Practitioners' knowledge, practices, and obstacles in the diagnosis and management of dementia. Aging Ment Health 2015; 19 (10): 912-20. 
5. O’Neill S, Richly P, Prats M, Mastadueno R, Pontello N, Christie C, et al. Diagnosis and Management of Cognitive Disorders in Latin America: New Technologies or more Knowledge Needed?. Poster session presented at: Alzheimer's Association International Conference (AAIC); 2013 July 13-18; Boston MA, U.S.A.

6. Marcano Belisario JS, Tudor KI, Sumalinog ARN, Middleton LT, Car J. Educational interventions for improving the skills of medical practitioners to detect, diagnose, and manage people with cognitive impairment and dementia (Protocol). Cochrane Database of Systematic Reviews 2013, Issue 6. Art. No.: CD010580. DOI: 10.1002/14651858.CD010580.
Apoyo Financiero: Fondecyt Conicyt / Fondecyt Regular / 1140423 (para CD y AS), Conicyt / Fondap/ 15150012 (para AS) \& Centro Basal para Centros de Excelencia, Proyectot FB 0003 de Programa Investigación Asociativa CONICYT (para AS).

\section{Correspondencia a:}

Dra. Andrea Slachevsky Ch.

Centro FONDAP Centro de Gerociencias. Salud Mental y Metabolismo (GERO).

Av. Salvador 486, Providencia, Santiago, Chile

Fono: (56-2) 29770530

Fono Fax: (56 2) 22741628

aslachevsky@me.com 